\title{
Perfil del informador religioso especializado en el Vaticano
}

\section{Miriam Diez-Bosch ${ }^{1}$}

Recibido: 2013-08-18

Enviado a pares: $2013-10-28$
Aprobado por pares: 2014-06-30

Aceptado: 2014-07-22

DOI: 10.5294/pacla.2015.18.1.11

Para citar este artículo / To reference this article / Para citar este artigo

Diez-Bosch, M. Marzo de 2015. Perfil del informador religioso especializado en el Vatica-

no. Palabra Clave 18(1), 258-275. D0I: 10.5294/pacla.2015.18.1.11

\section{Resumen}

La información religiosa especializada en el Vaticano tiene unas características propias que la distinguen de otras áreas y, al mismo tiempo, conlleva exigencias hacia la institución que cubre, la Santa Sede, que implican mayor flujo comunicativo y celeridad en la información. Este artículo analiza a los llamados vaticanistas, que además de cumplir las condiciones de ser acreditados ante la oficina de prensa de la Santa Sede, y vivir o frecuentar Roma, son un tipo de profesional periodístico particular. Después de describir los distintos perfiles, se añade la respuesta institucional vaticana a algunas de las peticiones, lagunas o sugerencias de los vaticanistas para el mejor desempeño de su labor.

\section{Palabras clave}

Religión, instituciones religiosas, catolicismo, periodismo, Santa Sede. (Fuente: Tesauro de la Unesco).

1 Doctora en Ciencias Sociales por la Pontificia Universidad Gregoriana. Profesora de la Facultad de Ciencias de la Comunicación. Universidad Ramon Llull. España. miriamdb@blanquerna.url.edu 


\section{Profile of a Religious Reporter Specialized in the Vatican}

\section{Abstract}

Religious information focused on the Vatican has no particular characteristics that distinguish it from news in other areas. Yet, covering the Holy See does entail certain demands that imply an enhanced flow of communication and timely reporting. This article analyzes the so-called Vaticanologists or Vatican experts, a particular breed of professional journalists who are accredited to the Press Office of the Holy See and are based in Rome or travel there often. A description of the various profiles is provided, followed by Vatican's institutional response to some of the requests, gaps or suggestions voiced by Vaticanologists to improve their reporting.

\section{Keywords}

Religion, religious institutions, Catholicism, journalism, Holy See (Source: Unesco Thesaurus). 


\section{Perfil do repórter religioso especializado no vaticano}

\section{Resumo}

A informação religiosa especializada no Vaticano tem características próprias que a diferenciam de outras áreas e, ao mesmo tempo, acarreta exigências da instituição que cobre, a Santa Sede, que implicam maior fluxo comunicativo e brevidade na informação. Este artigo analisa os chamados "vaticanistas", que, além de cumprirem as condições de serem acreditados ante a oficina de imprensa da Santa Sede e viver ou frequentar Roma, são um tipo de profissional jornalístico particular. Depois de descrever os diferentes perfis, adiciona-se a resposta institucional a algumas das petições, lagunas ou sugestões dos vaticanistas para o melhor desempenho de seu ofício.

\section{Palavras-chave}

Religião, instituições religiosas, catolicismo, jornalismo, Santa Sede (Fonte: Tesauro da Unesco). 


\section{Introducción}

Preguntas inoportunas, comentarios impertinentes y falta de formación y de sensibilidad. A los periodistas especializados en información religiosa, concretamente vaticana, se les acusa a menudo de estar poco preparados, de ser poco delicados, de no tener conocimientos teológicos, de politizar el tema religioso o de aplicar solo categorías mundanas a asuntos trascendentes. Ante estas habituales acusaciones, es necesario preguntarse qué necesita el informador religioso de la Iglesia, cuáles son las características que le deberían configurar y qué espera de una institución cuyo mensaje, a veces, no logra llegar al ágora pública.

Para abordar estas dificultades se apuntarán algunas ideas sobre la información religiosa en un contexto secularizado, además de trazar el perfil del llamado "vaticanista" o periodista especializado en religión católica. Esta investigación parte de la hipótesis de que ha habido una progresiva profesionalización de la información religiosa especializada en el Vaticano aunque, paradójicamente, la calidad de la información no siempre ha ido aumentando. Este estudio se basa en la observación participante en la sala de prensa de la Santa Sede y en el seguimiento de los llamados vaticanistas, que en esta investigación se dividen en varias tipologías, según el tipo de medio para el que trabajan. Todos los ejemplos se refieren a perio$\operatorname{distas}^{2}$ que trabajan en el Vaticano o se desplazan a menudo a la Santa Sede y, por tanto, tienen una relación fluida con las fuentes y con la misma sala stampa u oficina de prensa de la Santa Sede.

\section{Perfil del vaticanista}

Los hay de varios tipos. Veteranos cultísimos y recién llegados sin más conocimiento que el epidérmico barniz que proporcionan las noticias religiosas en los medios generalistas. Algunos son agresivos, incisivos y directos, al estilo de la tajante periodista italiana Oriana Fallaci (Florencia, 1929-2006). Otros, discretos y repletos de contactos secretísimos. Algunos llegan a vaticanistas casi como un castigo: se ven despojados de su especialización periodística en política y les asignan seguir la aparentemente gris vida vaticana.

2 Y como tales cumplen con las características personales y profesionales que indica Randall (2000). 
Todos ellos tienen algunos elementos en común. Pasión por el intrigante mundo vaticano. Paciencia para adentrarse en los entresijos de una de las instituciones más complejas del mundo. Tacto y olfato periodístico para detectar dónde reside la noticia. Se les puede ver en las librerías ojeando el último panfleto antivaticano de turno, o conversando con un joven monseñor en una zona reservada de las pizzerías más concurridas. Es posible cruzarse con ellos en un distinguido restaurante de la Apia con un embajador, y siguiendo a los devotos en un rosario de jóvenes. Se han convertido no solo en informadores, sino en protagonistas de la información vaticana, y no es difícil adivinarlos de estrellas invitadas en una tertulia radiofónica. Lo suyo son las fuentes: ellos mismos se convierten en fuente (Rodríguez, 2001). Son los vaticanistas, los informadores religiosos especializados en la Santa Sede. Su lugar natural es Roma. ${ }^{3}$ Su nombre, periodistas. Su apellido, vaticanistas. Estos seres no son ángeles, aun viviendo rodeados de un ambiente divino. Uno de los vicios que pueden salpicar a los vaticanistas es la conducta vampirista: abusar o aprovecharse de los demás, sean estos también periodistas o fuentes varias. Y lo peor que le puede ocurrir a un informador del Vaticano es perder la credibilidad. La credibilidad puede peligrar por comentarios de colegas que desautoricen una fuente no contrastada, una tendencia obsesiva hacia el rumor y la invención. La reputación del vaticanista se gana con una vida, y se puede perder con un simple y desenfocado desliz. ${ }^{4}$

\section{El origen del término}

La figura de los informadores religiosos sobre el Vaticano ha adquirido, en las últimas décadas, un perfil cada vez mejor trazado, y es ya una tradición considerarlos no periodistas religiosos sino vaticanistas. La cobertura de la actividad de la Santa Sede se remonta en tiempos modernos a 1929, con los Pactos de Letrán. En este momento se intensificó la atención mediática por la Santa Sede.

3 Dice Riccardi (en Zizola, 2002): "La informacion religiosa viene acompañada por una red de contactos, de amistades, de que solo se pueden desenvolver en este terreno tan particular que es Roma (...). Para un vaticanista, distanciamento o participación son dos polos en los que difícilmente se encuentra un punto medio. Son precisamente estos extremos del tejido de Roma, la capital italiana, la ciudad del Papa, el ágora de tantos encuentros religiosos y culturales, terreno de experiencias humanas".

4 Basta recordar el escándalo de los Vatileaks y cómo la información en el Vaticano se ha convertido en material vulnerable y a merced de los anónimos. 
Zizola (1996) explica que a principios de febrero del año 1939, cuando Pío XI estaba a punto de fallecer, los periodistas fueron encerrados en llave en una angosta sala en el patio de San Dámaso, dentro del Vaticano. Era el lugar para congresos y trabajo. Tenían pocos teléfonos, poquísima libertad, pero la ventaja de poder controlar desde las ventanas a los cardenales que salían al palacio apostólico para las audiencias papales. No había más noticias: quien se ocupaba del Vaticano lo hacía confiando en sus propios amigos, interpretando, moviéndose en ambientes curiales, poniendo todo su empeño en descifrar. Descifrar el Vaticano ha sido, y continúa siendo, la principal misión de los informadores vaticanos, que no solo cuentan qué ocurre sino por qué se dan algunas circunstancias que sin un contexto serían imposibles de comprender. En un mundo en el que la frontera entre rumor y noticia no es objetiva (Argemí, 2013), es preciso tener en cuenta que hasta los rumores más mentirosos tienen su público, y hay verdades que ciertos públicos nunca percibirán como tales porque resulta más cómodo tirar de rumores, guardaespaldas eficaces de sus verdades subjetivas. En el campo vaticano, el rumor se instala con mucha facilidad, a veces por falta de fuentes directas $y$, por tanto, por la dificultad misma de ir a la fuente; en otras ocasiones, porque es la estrategia comunicativa que se expande rápido, crea confusión e interés y despierta atención hacia temas altamente sensibles e interpretables. Además, la información religiosa se ve, no pocas veces, marginada por clichés (Díez, 2006), lo que dificulta todavía más su necesaria naturalización en los medios.

Relata Kairu (2003), ${ }^{5}$ en su estudio sobre los vaticanistas, que el término italiano vaticanista se ha impuesto ante el inglés vaticanologist, ylo define como el experto que cubre noticias religiosas: el reportero vaticano se encuentra a sí mismo cubriendo el contexto que abraza a la Ciudad del Vaticano y las actividades de la Santa Sede. Para calificarlo así, tiene que tener una acreditación en la oficina de prensa de la Santa Sede. Este background de conocimiento hace posible describir al vaticanista como un periodista acreditado ante la Santa Sede que se ocupa de cubrir las noticias de la Ciudad del Vaticano y de la Santa Sede. El vaticanista es un experto en

5 El suyo es el primer estudio que analiza de manera académica la tipología del informador religioso vaticanista. 
la cobertura de información religiosa que concierne al gobierno eclesiástico y a distintos aspectos de la iglesia católica.

Este vaticanista posee, en principio, competencias en los distintos aspectos que envuelven la esencia, identidad y organización de la iglesia católica en general y del mundo vaticano en particular. Kairu distingue entre dos tipos de vaticanistas: los que se ocupan de cubrir exclusivamente noticias de la Santa Sede, que suelen ser italianos, residentes en Roma y asiduos de la oficina de prensa de la Santa Sede, y los otros, que tienen que cubrir Santa Sede pero también Italia para sus medios respectivos.

Las noticias importantes para la Iglesia, en algunos casos, son cubiertas por personas que no son especialistas en religión, y esto puede tener importantes consecuencias prácticas (Shaw, 1999).

\section{El vaticanista que trabaja para un medio confesional}

Este primer perfil es el más común. Se trata del experto en información religiosa vaticana cuya dedicación exclusiva o mayoritaria está concentrada en un medio confesional. Sería el caso del corresponsal en Roma del periódico católico francés La Croix, por ejemplo, o de los periodistas que trabajan en el Osservatore Romano, o el periódico de la Conferencia Episcopal Italiana Avvenire. A estos se unen periodistas que trabajan para medios como agencias especializadas en religión, como la Agencia Internacional Zenit, el Sir, Aciprensa o la Agencia Catholic News Service, de los Estados Unidos. Estos periodistas están consagrados a un tipo de información sectorial muy acotada. Sus colegas en el mismo medio, que se ocupan de secciones no estrictamente consideradas religiosas (espectáculos, internacional), pueden no ser llamados "vaticanistas", y en varias ocasiones rechazan esta etiqueta. Es decir, un periodista que trabaje en un periódico confesional no es automáticamente un vaticanista. $Y$ un vaticanista puede trabajar en un medio confesional o en un medio laico. Su área de expertise en materia religiosa es lo que lo define, no su medio, ni su fe o su falta de ella (podríamos hablar de vaticanistas no creyentes, ya que el vaticanista, como hemos visto, es el profesional especializado en información religiosa vaticana, independientemente de su creencia religiosa personal). Los acreditados permanentemen- 
te en Roma forman parte, si lo desean, de la Associazione Internazionale Giornalisti Accreditati in Vaticano o Aigav.

\section{El vaticanista que trabaja en un medio laico}

Es el experto en religión (mundo vaticano especialmente) en un medio no confesional. Los hay veteranos, como Marco Politi en Il Fatto Quotidiano o Luigi Accattoli en Il Corriere della Sera. Sus medios les han contratado para dedicarse exclusivamente al seguimiento en profundidad del tema vaticano. Son los puntos de referencia para los nuevos vaticanistas que se incorporan a la profesión. Algunos son periodistas consagrados y reconocidos en su ámbito, como Phil Pullella, de la Agencia Reuters. Otros trabajan desde la periferia no romana, pues no son corresponsales en sentido estricto sino especialistas que siguen viviendo en sus lugares de trabajo, como durante años hizo Henri Tinq para Le Monde en París o Juan Manuel Vidal, desde Madrid, para El Mundo. Cabe mencionar también a periodistas como Andrés Beltramo, de Notimex y Vatican Insider, Andrea Tornielli, de La Stampa, Franca Giansoldati, de Il Messaggero o Giovanna Chirri, de Ansa, todos ellos basados en Roma. Uno de los más conocidos a nivel internacional es John L. Allen, periodista vaticanista del Boston Globe y ahora editor del portal Crux.

El 11 de Septiembre de 2001 (9/11) fue un catalizador para la información religiosa. Como señala Hoover (2012), los ataques fueron un punto de inflexión en la historia, y fueron vividos de modo profundo porque fueron mediados en tiempo real. Si bien su relación con la religión quizá es menos obvia, la religión ha ido cobrando cada vez más importancia y se ha evidenciado como una dimensión importante en la historia. Es en este momento en el que muchos editores se dieron cuenta de que no contaban, en sus plantillas, con especialistas en religión. Proliferaron entonces los cursos a nivel universitario, publicaciones, recursos, para una información religiosa de calidad. En la elección del papa Francisco, en marzo de 2013, no faltaron especialistas de Al-Jazeera, $\mathrm{CNN}$ y otras grandes cadenas globales para cubrir de manera eficaz el evento. ${ }^{6}$

6 Ya después de la celebración del Concilio Vaticano II empezaron a resonar reflexiones sobre la necesidad de una mayor transparencia informativa en la Iglesia (Remy, 1971). 
Es cada vez más evidente que la fe religiosa tiene una proyección social y existen razones para una apertura de los medios al mensaje religioso, vista la función de las confesiones religiosas como integrantes de la sociedad civil (Martí, 2003).

\section{El laico que trabaja en un medio confesional}

Podríamos establecer otra tipología, que sería la del periodista que trabaja para un medio confesional y realiza en él tanto informaciones específicamente religiosas, como otras más culturales y menos explícitas referidas al campo espiritual o religioso. Nos referimos al profesional que trabaja, por ejemplo, en la revista Famiglia Cristiana, en Italia, pero se ocupa de moda o de deportes. Su interés personal y profesional no es el religioso, pero su medio lo es o el medio pertenece a un grupo religioso, con lo cual, la cosmovisión del medio está, aunque no explícitamente, enmarcada en la fe. Revistas generalistas como la mencionada no se encuentran en otros contextos. Famiglia Cristiana ha llegado a tiradas de un millón de ejemplares en sus momentos más álgidos. El periodista laico que se ocupa de temas varios -no religiosos- en un medio confesional es también aquel por ejemplo que cubre moda o política en una radio como la española COPE. Su interés por la información religiosa, en este sentido, puede ser mínimo, pues no se trata de un campo que le toque cubrir en su quehacer cotidiano.

\section{El católico que trabaja en un medio laico}

Es el caso de los periodistas que ejercen de portavoces de lo eclesial en medios laicos, especialmente en el ámbito de la opinión y no tanto en informaciones, aunque no se descartan. En este sentido, podríamos referirnos a las firmas que algunos periódicos laicos suelen atraer, cuando invitan a intelectuales católicos a escribir en grandes periódicos como El País, International Herald Tribune o La Repubblica. Cada vez más, los medios incluyen voces religiosas plurales en sus parrillas. A veces, simplemente para generar debate y polémica con enfrentamientos. En otras ocasiones, se trata de colaboraciones de católicos que tienen un espacio para un artículo o una intervención en los medios audiovisuales. En este sentido, cabe citar la iniciativa de Catholic Voices a nivel mundial, que intenta situar en el ágora 
generalista mediática -especialmente televisiva- voces de católicos, normalmente no eclesiásticos.

\section{El "otro" vaticanista: identidad transversal}

Existe un periodista todoterreno que ni trabaja para un medio confesional solamente, ni se ocupa quizá solo de temas vaticanos, y cuya identidad religiosa es desconocida o ambigua. Son los "otros vaticanistas", los de identidad transversal. Son periodistas versátiles, que quizá se encargan de temas vaticanos a corto plazo, vaticanistas a tiempo determinado. También podrían encajar los no creyentes, que al escribir sobre temas religiosos, se acaban convirtiendo en los -paradójicamente- defensores de la ortodoxia católica. Articulistas que en sus escritos defienden o se alinean con posiciones próximas a la Iglesia, pero que no provienen del periodismo (escritores, abogados, profesores). Aquí también cabe lo que Pou Amérigo (2008) ${ }^{7}$ define como la diferencia entre el profesional y el activista: la visión "profesional" considera que la información religiosa es un contenido periodístico más, que requiere un tratamiento específico derivado de su especialización y que exige el mismo rigor y las mismas pautas de conducta profesional que las demás secciones. En cambio la visión "activista" es la que asimila información religiosa y difusión de la fe, porque considera que el periodismo religioso, por ejemplo en el contexto católico, ha de compartir los fines de la Iglesia y estar al servicio de la información.

Estos perfiles de vaticanista tienen en común una característica: se lamentan de lo críptica que a menudo resulta la información vaticana y lo complicado que resulta contar con informaciones en tiempos breves y en un lenguaje claro.

\section{La irrupción de los bloggers}

Antes de la expansión de las redes sociales, los bloggers especializados en religión han ocupado parte del espacio que antes solo ostentaban los periodistas reconocidos como vaticanistas. No en vano, el mismo Vaticano se ha

7 En su reflexión sobre la información religiosa, Pou Amérigo aclara que este tipo de periodismo especializado tiene peculiaridades que lo distinguen de otras áreas de especialización. 
interesado por el fenómeno, y organizó, en Roma, el primer encuentro de bloggers católicos del mundo (Vatican Meeting for Bloggers), en el 2011.

En la blogosfera crece el número de articulistas que opinan sobre temas religiosos e informan sobre el Vaticano, y algunos de ellos se convierten en auténticos líderes de opinión. Fuera del cerco estrictamente vaticano y en el campo del catolicismo más general, solo en lengua española podemos citar blogs como Blog B16-links, Blogueros con el Papa y los bloggers albergados en portales como Religión en Libertad, Religión Confidencial, Infocatólica, Religión Digital o el ya citado Vatican Insider. Uno de los aspectos que modifican hoy la información religiosa es precisamente esta proliferación de voces ciudadanas que se añaden a los especialistas. En algunos casos, impulsan a la información vaticana a ser todavía más rápida y precisa. Ciertos bloggers ejercen de cómplices de los vaticanistas, ofreciéndoles información o pistas para profundizar en algunos asuntos. Otros juegan a despistar y desprestigiar. Tanto unos como otros son termómetros en la fluidez de la información relativa al universo religioso, concretamente acerca del Vaticano.

\section{Quejas por parte de los periodistas}

Una queja recurrente son las respuestas tardías, incompletas, previsibles que ofrece la fuente eclesial. Que las respuestas vaticanas no sean periodísticas no sería en sí un defecto a achacar a la institución: para esto están los periodistas. Es su cometido convertir declaraciones en información. Si bien no se puede pedir que todo lo que emane del Vaticano sea, en sí mismo, información en forma de píldora informativa, sí que parece sensato pedir que las reacciones, declaraciones, comunicados y respuestas lleguen a tiempo, contengan la información necesaria y aporten alguna novedad, datos que no sean previsibles ni repetitivos. Esto no siempre es así: en varias ocasiones, los periodistas que cubren el Vaticano podrían haber escrito una noticia antes de una rueda de prensa, y los datos con los que contaban eran ya precisos, pues la rueda les ha simplemente aportado la lectura, en voz alta, de ellos.

Otra demanda que realizan los informadores vaticanos es la de la disponibilidad y la respuesta. Disponibilidad de espacios y de tiempos, que 
se tengan en cuenta las necesidades logísticas y temporales de los periodistas. El espacio en la sala de prensa, fundada el 16 de octubre de 1966 por iniciativa de Pablo VI, en la Via della Conciliazione en Roma, es realmente exiguo para la cantidad de reporteros, especialmente en momentos clave. La institución realiza esfuerzos notables en momentos de máxima atención mediática, como la elección del Papa o canonizaciones. El horario de mañana tampoco ayuda a los periodistas de otras latitudes a estar conectados con la Santa Sede. También señalan una falta de respuesta ágil y fácil, no vaga ni excesivamente dilatada en el tiempo por parte de la oficina que comunica a los periodistas las múltiples actividades de la Santa Sede, especialmente en lo que concierne a la agenda papal. Y un aspecto no menos importante, piden que se puedan atender las demandas para viajar en el avión papal y que no recaigan siempre sobre los mismos veteranos, aspecto este difícil de armonizar con un aluvión general de peticiones (la media de acreditados permanentes es de 350). El avión cuenta con espacios reducidos. Y desde la institución se promueve la política de priorizar a las agencias antes que a los periódicos o radios para intentar llegar a cuanta más gente sea posible.

\section{La articulada respuesta institucional vaticana}

Para paliar estas quejas, la Santa Sede ha activado una respuesta articulada.

1. Sinergia vaticana: la Santa Sede ha aunado esfuerzos para que todos los dispositivos informativos, organismos de comunicación y medios propios confluyan y estén más coordinados. El acercamiento entre varios servicios, desde digitales a la radio, pasando por la oficina de información (sala stampa) y por la televisión, han llevado a una convergencia que desemboca en la creación de un portal renovado multimedia, el portal news.va. La Santa Sede quiere optimizar esfuerzos y evitar pérdidas en el terreno comunicativo. Aun con déficit económico, es un ámbito del que no va a prescindir por ser connatural a la nueva evangelización. Esta unidad permite que el informador vaticano tenga más clara la estructura institucional, los canales y soportes. 
2. Amplitud de servicios ${ }^{8}$ digitales: Youtube ha significado un salto en la respuesta vaticana a la comunicación. El hecho de disponer diariamente de videos del Papa en esta plataforma, así como de los videos del Centro Televisivo Vaticano, en nueve idiomas, es uno de los mayores esfuerzos audiovisuales multimedia nunca realizados por la iglesia católica. Además, la página web vaticana oficial (www.vatican. va) está constantemente incrementando sus servicios y entrando en una mentalidad más participativa, que la ha llevado no solo a crear news.va, sino también perfiles en las redes sociales. Solo mencionando Twitter, tenemos el perfil oficial del Papa, @pontifex, el de la Secretaría de Estado, @terzaloggia, o el de la oficina de celebraciones litúrgicas del Sumo Pontefice, @ucepo. Los actos en streaming y el esfuerzo multimedia son notables en los últimos tiempos y se han visto acelerados con la elección del papa Francisco.

3. Atención más personalizada: una de las prioridades en las nuevas tendencias informativas es la de contrarrestar el anonimato que la globalización ha acarreado. En la tarea de la relación con los medios, las oficinas de prensa difunden mensajes que tienen que ser, en palabras de Carroggio (2009: 247), "verdaderos, cargados de empatía, claros y socialmente responsables". Desde el Vaticano se intenta conocer a los vaticanistas, atender sus peticiones personalmente y no solo en bloque. En un entorno en que prevalecen más las relaciones y el intercambio que los contenidos mismos (Spadaro, 2012), esto es más apremiante que nunca.

4. Prioridad a las agencias: sin desdeñar a ningún medio de comunicación, la Santa Sede como institución privilegia el contacto con las agencias de noticias (Reuters, AP, Efe, Ansa...) para que sean ellas las difusoras primeras de los comunicados oficiales. El cuidado a los periodistas de agencia se entiende desde esta óptica de llegar a cuanta más población sea posible. Y sobre todo más rápido: en el mundo del periodismo es importante llegar antes que la competencia, aunque el público premia más la calidad que la celeridad (Contreras, 2001).

8 En este sentido sería orientador seguir las indicaciones de Daniel Arasa en su modelo de análisis sobre la comunicación de la Iglesia a través de las webs diocesanas, y ver de qué manera los vaticanistas establecen una relación con la web vaticana totalmente distinta de la que se teje con sus diócesis de origen (cfr. Arasa, 2008). 
5. Descentralización de los mensajes y apertura a lo local: la Santa Sede se apoya en las iniciativas y buenas prácticas locales. Incentiva por ejemplo y anima ideas como la publicación How to Cover the Catholic Church, de la oficina de medios de la Conferencia Episcopal de los Estados Unidos, o los glosarios religiosos de conferencias como las de Chile o España.

Esta respuesta vaticana parte de una premisa, y es que la lógica del Evangelio no coincide con la lógica del mundo. Los valores que la Iglesia intenta transmitir (amor, perdón, servicio, aceptación de la cruz) no son los mitos de poder, superación individual, imperio, orgullo y venganza que imperan en la sociedad, tal y como subraya Robert A. White (1997). Pero la Iglesia siente el deber de atender a los medios, y de informar correctamente, porque en ello se juega su misión. Para conseguirlo, el esfuerzo de adaptación a un nuevo mensaje sin perder el simbolismo de la tradición es un desafío.

Juan Díaz-Bernardo (2002) lo especifica así:

La recuperación del universo simbólico, en la capacidad creadora de la palabra y el poder evocador de la imagen, brindan nuevas posibilidades en la regeneración de un lenguaje capaz de proyectar en las culturas unos resortes adecuados para la transformación de sus modos de percepción, personal y comunitaria, de la existencia y de la presencia de lo sagrado.

\section{Conclusión}

La fe religiosa se expresa en el contexto público y los medios no pueden obviarla como hecho noticioso. Este es uno de los motivos que explican la proliferación de periodistas especializados en religión. Sin embargo, no siempre esta mayor cantidad de profesionales es, automáticamente, sinónimo de excelencia en la información. Hay varios factores que dificultan esta calidad: por una parte, la crisis en el sector no siempre consiente que exista un profesional dedicado exclusivamente al tema. Otro es la rotación: si antaño podíamos perfilar a vaticanistas con incluso tres décadas de dedicación, hoy es más habitual que no sobrepasen una década de especialización 
y se dediquen a otros temas por exigencias de sus medios. El periodismo ciudadano también se ha infiltrado en el horizonte de la especialización religiosa, y si bien sus aportaciones son, en muchos casos, inéditas y valiosas, puede contaminar la jerarquía de la información y crear confusión entre rumores y buen periodismo.

Lo que los vaticanistas piden a la Iglesia es que esta, como institución, sea ella misma y comunique su identidad. Los vaticanistas, por su parte, antes que vaticanistas son periodistas, y este factor comporta que quizá respetan la fe, aunque tal vez no la compartan, en la vida de las personas. Este respeto se le exige al periodista que informa sobre religión, puesto que la burla o una actitud de recelo constante sería peligrosa y poco contrastada. El vaticanista tiene una curiosidad inmensa sobre religión y espiritualidad y, en general, los informadores sobre religión poseen un alto sentido de la justicia. No se conforman con una fuente porque no quieren ser instrumentalizados, ni tampoco presionan a favor del catolicismo, pretendiendo que este salga con una "buena luz", sino que ofrecen sus informaciones lo más veraces y equilibradas posible, también en aquello negativo. No ridiculizan a las otras religiones y si es preciso se asesoran con veteranos. Otra de sus características es que atribuyen explicaciones sin implicarse ni defender causas.

Los vaticanistas están cambiando. Ya no son solo veteranos, el perfil es joven y rota a menudo. No es tan habitual ya encontrar gente con décadas de experiencia, ya que la movilidad actual no lo propicia. Los vaticanistas piden a la Iglesia profesionalidad, respeto a sus tiempos y claridad. Solicitan que la Iglesia se adecúe a sus tiempos, y no es una exigencia desproporcionada, sino realista. Si el periodismo es inmediatez, la Iglesia es eternidad. Pero entre ambas puede mediar un terreno de entendimiento, en beneficio mutuo.

La Iglesia pide a los vaticanistas delicadeza, profesionalidad, sensibilidad, finezza y competencia. Los vaticanistas empujan a la Iglesia con sus demandas a una mayor claridad y fluidez en la información. Para ello es preciso un mejor flujo de información -basado en la confianza interna-, una mayor competencia mezclada con savoir faire en el tacto y la relación con 
los periodistas y una credibilidad y claridad estratégica que vincule más evangelización con medios de comunicación, pues no son dos mundos antagónicos y los recientes esfuerzos en las sinergias vaticanas en materia de comunicación lo corroboran.

A veces, el conocimiento general que tiene el periodista es un mezcla de información, desinformación y prejuicios. Sin embargo, es preciso notar que los vaticanistas acreditados ante la Santa Sede no son una raza de periodistas desinformados. Su peculiaridad hace de ellos un sector que conserva el prestigio del buen periodismo, aquel que va a la búsqueda de la verdad, que es constante, que cultiva las fuentes y tiene respeto por su público.

\section{Referencias}

Arasa, D. (2008). Church communications through diocesan websites. Roma: Edusc.

Argemí, M. (2013). Rumors en guerra. Desinformació, internet i periodisme. Barcelona: A contra vent.

Carroggio, M. (2009). L’ufficcio stampa e la relazione con i media. En La Porte, J. M. (Ed.), Introduzione alla Comunicazione istituzionale della Chiesa. Roma: Edusc.

Contreras, D. (2001). Il linguaggio giornalistico: caratteristiche e limiti. En Stenico, T. (Ed.), Era mediatica e nuova evangelizzazione (4965). Vaticano: LEV.

Díaz-Bernardo, J. (2002). Anunciar a Cristo en la aldea global. La Iglesia en la sociedad de la información (182). Toledo: Estudio Teológico de San Ildefonso.

Díez, M. (2006). La información religiosa. Hacia una naturalización del periodismo religioso en los medios. En Srampickal, J., Mazza, G. \& Baugh, L. (Eds.), CrossConnections, interdisciplinary communication 
studies at the gregorian university (67-76). Roma: Editrice Pontificia Università Gregoriana.

Filteau, J. \& Cipolla, B (Eds.) (2008). How to cover the Catholic Church. Washington D. C.: United States Conference of Catholic Bishops.

Hoover, S. M. (2012). Religion, the media and 9/11. En Gordon, L., Jolyon, M. \& Strhan, A. (Eds.). Religion, media and culture: A reader (75-88). Londres-Nueva York: Routledge.

Kairu, F. N. (2003). The vaticanologists: Who they are and what they do. A survey on their knowledge, attitudes and behaviour. Roma: Edusc.

Martí, J. M. (2003). Sociedad, medios de comunicación y factor religioso. Perspectiva jurídica, (99). Cuenca: Alfonsípolis.

Pou Amérigo, M. J. (2008). El hecho religioso y su tratamiento periodístico: limitaciones y dificultades. Estudios sobre el Mensaje Periodístico, 14, 561-573.

Randall, D. (2000). The universal journalist. Londres: Pluto Press.

Remy, J. (1971). The diffusion of information in the church: A way out of the unequal dialogue? Concilium, 3(7), 108-116.

Rodríguez, G. M. (2001). ¿Quién habla en nombre de la Iglesia? El anunciador del mensaje evangélico en el magisterio de la Iglesia. Religión y Cultura, XLVII, 117-141.

Shaw, R. (1999). Public relations in the Church. En Shaw, R., Mammoser, T. L. \& Maniscalco, F. (Eds.), Dealing with media for the Church (79). Roma: Edusc.

Spadaro, A. (2012). Cyberteologia. Pensare il cristianesimo al tempo della rete. Milán: Vita e Pensiero. 
White, R. A. (1997). Los medios masivos y la evangelización. En Borobio, D. \& Ramos, J. (Eds.), Evangelización y medios de comunicación (95). Salamanca: Universidad Pontificia de Salamanca, Bibliotecha Salmanticensis, Estudios 186.

Wolton, D. (1999). L'Église et les deux défis de la société de l'information et de l'Europe. En CEEM, Evangelio, Iglesia y sociedad de la información (43-50). Madrid: Edice.

Zizola, G. (1996). La Chiesa nei media. Torino: Società Editrie Internazionale.

Zizola, G. (2002). L'informazione in Vaticano. Da Pio IX a Giovanni Paolo II. Villa Verucchio: Pazzini Editore. 